Bull. Korean Math. Soc. 51 (2014), No. 4, pp. 1155-1161

http://dx.doi.org/10.4134/BKMS.2014.51.4.1155

\title{
THE $q$-DEFORMED GAMMA FUNCTION AND $q$-DEFORMED POLYGAMMA FUNCTION
}

\author{
Won Sang Chung, Taekyun Kim, and Toufik Mansour
}

\begin{abstract}
In this paper, we rederive the identity $\Gamma_{q}(x) \Gamma_{q}(1-x)=$ $\frac{\pi_{q}}{\sin _{q}\left(\pi_{q} x\right)}$. Then, we give $q$-analogue of Gauss' multiplication formula and study representation of $q$-oscillator algebra in terms of the $q$-factorial polynomials.
\end{abstract}

\section{Introduction}

In the last decades, the $q$-calculus served as a bridge between mathematics and physics. The majority of researchers around the world who use $q$-calculus are physicists. This field has expanded explosively, due to the fact that the basic hypergeometric series served several subjects of combinatorics, quantum theory, number theory, statistical mechanics.

From now on we will restrict our concern to the case that the deformation parameter $q$ is real and $0<q<1$. The $q$-analogue of Gamma function (or $q$-deformed Gamma function), a $q$-analogue of Euler's gamma function, was introduced by Thomae [17] and later by Jackson $[5,6]$ as the infinite product

$$
\Gamma_{q}(x)=(1-q)^{1-x} \prod_{n=0}^{\infty} \frac{1-q^{n+1}}{1-q^{n+x}} .
$$

Askey [2] pointed out that this function satisfies $\Gamma_{q}(x+1)=[x] \Gamma_{q}(x), \Gamma_{q}(1)=1$ and $\frac{d^{2}}{d x^{2}} \log \Gamma_{q}(x+1)>0$ for all $x \geq 0$, where $[x]$ is a Jackson's $q$-number defined by $[x]=[x]_{q}=\frac{1-q^{x}}{1-q}$. Recently, several authors have studied many properties of $q$-gamma functions $\Gamma_{q}(x)$ in the area of physics and applied mathematics (see $[1,3,8,7,9,10])$. In this paper, we are interested on $q$-deformed Gamma and polygamma functions. The aim of this paper is to rederive the identity $\Gamma_{q}(x) \Gamma_{q}(1-x)=\frac{\pi_{q}}{\sin _{q}\left(\pi_{q} x\right)}$. At the end, we give $q$-analogue of Gauss' multiplication formula and study representation of $q$-oscillator algebra in terms of the $q$-factorial polynomials.

Received September 19, 2013; Revised January 22, 2014.

2010 Mathematics Subject Classification. 11B68, 33D05, 11B65.

Key words and phrases. q-gamma function, $q$-polygamma function. 


\section{New properties concerning the $q$-deformed Gamma function and $q$-deformed polygamma function}

The $q$-deformed Gamma function can be rewritten in the following form;

$$
\Gamma_{q}(x)=\lim _{n \rightarrow \infty} \frac{[n] ![n]^{x}}{[x][x+1][x+2] \cdots[x+n]},
$$

where the $q$-factorial is defined by $[n] !=[n][n-1] \cdots[2][1]$. For an integer $N$, we have $\Gamma_{q}(N)=[N-1]$ ! The proof is as follows.

$$
\begin{aligned}
\Gamma_{q}(N) & =\lim _{n \rightarrow \infty} \frac{[n] ![n]^{N}}{[N][N+1][N+2] \cdots[N+n]} \\
& =[N-1] ! \lim _{n \rightarrow \infty} \frac{[n]^{N}}{[n+1][n+2] \cdots[n+N]}=[N-1] !,
\end{aligned}
$$

where we used $\lim _{n \rightarrow \infty}[n+k]=\frac{1}{1-q}$. Using (1), we obtain the duplication formula of the $q$-deformed Gamma function.

Proposition 2.1. The q-Gamma function obeys

$$
\Gamma_{q}(x) \Gamma_{q}\left(x+\frac{1}{2}\right)=\Gamma_{q}\left(\frac{1}{2}\right)\left([2]_{\sqrt{q}}\right)^{1-2 x} \Gamma_{\sqrt{q}}(2 x),
$$

where $[x]_{\sqrt{q}}=\frac{1-(\sqrt{q})^{x}}{1-\sqrt{q}}$.

Proof. Using (1), we have

$\Gamma_{q}(x) \Gamma_{q}\left(x+\frac{1}{2}\right)=\lim _{n \rightarrow \infty} \frac{([n] !)^{2}[n]^{2 x+1 / 2}}{[x][x+1] \cdots[x+n][x+1 / 2][x+3 / 2] \cdots[x+n+1 / 2]}$.

Using $[2 x]_{\sqrt{q}}=[2]_{\sqrt{q}}[x]$, we have

$$
\begin{aligned}
\Gamma_{q}(x) \Gamma_{q}\left(x+\frac{1}{2}\right) & =\lim _{n \rightarrow \infty} \frac{\left([2]_{\sqrt{q}}\right)^{2 n+1}([n] !)^{2}\left([2]_{\sqrt{q}}\right)^{-2 x-1}\left([2 n]_{\sqrt{q}}\right)^{2 x+1 / 2}}{[2 x]_{\sqrt{q}}[2 x+1]_{\sqrt{q}} \cdots[2 x+2 n]_{\sqrt{q}}[2 x+2 n+1]_{\sqrt{q}}} \\
& =A\left([2]_{\sqrt{q}}\right)^{-2 x} \Gamma_{\sqrt{q}}(2 x) .
\end{aligned}
$$

Inserting $x=1 / 2$, we derive $A=[2]_{\sqrt{q}} \Gamma_{q}\left(\frac{1}{2}\right)$, which completes the proof.

Note that in [10] Mansour has also studied some properties of $\Gamma_{q}(x)$ related to our Proposition 2.1. In order to compute $\Gamma_{q}\left(\frac{1}{2}\right)$, let us introduce the $q$-deformed exponential function. There exist two different $q$-deformed exponential functions as follows:

$$
e_{q}(x)=\sum_{k=0}^{\infty} \frac{x^{k}}{[k] !}, E_{q}(x)=\sum_{k=0}^{\infty} \frac{q^{k(k-1) / 2} x^{k}}{[k] !} .
$$

The following relations exist between the two types of the $q$-deformed exponential functions;

$$
e_{q}(x)=E_{q^{-1}}(x), e_{q}(-x) E_{q}(x)=1
$$


For $0<q<1$, we have $e_{q}(-\infty)=0, e_{q}\left(\frac{1}{1-q}\right)=\infty, E_{q}\left(-\frac{1}{1-q}\right)=0$ and $E_{q}(\infty)=\infty$. Using the $q$-deformed exponential functions, we get the well known integral expression for the $q$-deformed Gamma function (see [6]):

$$
\Gamma_{q}(n)=\int_{0}^{\frac{1}{1-q}} x^{n-1} E_{q}(-q x) d_{q} x
$$

Therefore, we can state the following result.

Proposition 2.2. For $0<q<1$,

$$
\Gamma_{q}\left(\frac{1}{2}\right)=\sqrt{1-q} E_{q}\left(\frac{q}{q-1}\right) e_{q}\left(q^{1 / 2}(1-q)\right) .
$$

Proof. By (2) we have

$$
\begin{aligned}
\Gamma_{q}\left(\frac{1}{2}\right) & =\int_{0}^{\frac{1}{1-q}} x^{-1 / 2} E_{q}(-q x) d_{q} x \\
& =\sum_{n=0}^{\infty} q^{n}\left(\frac{q^{n}}{1-q}\right)^{-1 / 2} E_{q}\left(-\frac{q^{n+1}}{1-q}\right) \\
& =\sqrt{1-q} \sum_{n=0}^{\infty} q^{n / 2}\left(1-q^{n+1}\right)_{q}^{\infty} \\
& =\sqrt{1-q}\left(1-q^{n+1}\right)_{q}^{\infty} \sum_{n=0}^{\infty} \frac{q^{n / 2}}{[n] !}(1-q)^{n} \\
& =\sqrt{1-q} E_{q}\left(\frac{q}{q-1}\right) e_{q}\left(q^{1 / 2}(1-q)\right),
\end{aligned}
$$

where $(1+a)_{q}^{\infty}$ is defined as $\prod_{j \geq 0}\left(1+q^{j} a\right)$ and we used the formula

$$
\sum_{n \geq 0} q^{n / 2}\left(1-q^{n+1}\right)_{q}^{\infty}=e_{q}\left(q^{1 / 2}(1-q)\right)(1-q)_{q}^{\infty}
$$

The $q$-deformed Gamma function can be also expressed in the infinite product form:

$$
\frac{1}{\Gamma_{q}(x)}=[x] \prod_{k=1}^{\infty}\left[1+\frac{x}{k}\right]_{q^{k}}\left(\left[1+\frac{1}{k}\right]_{q^{k}}\right)^{-x},
$$

where $[x]_{q^{k}}=\frac{1-q^{k x}}{1-q^{k}}$. Using (3), we have

$$
\Gamma_{q}(x) \Gamma_{q}(1-x)=\frac{1}{[x] \prod_{k=1}^{\infty}\left[1+\frac{x}{k}\right]_{q^{k}}\left[1-\frac{x}{k}\right]_{q^{k}}} .
$$

By inserting $x=1 / 2$, we obtain the $q$-analogue of Wallis formula:

$$
\left(\Gamma_{q}\left(\frac{1}{2}\right)\right)^{2}=[2]_{\sqrt{q}} \cdot \frac{[2]_{\sqrt{q}}[2]_{\sqrt{q}}}{[1]_{\sqrt{q}}[3]_{\sqrt{q}}} \cdot \frac{[4]_{\sqrt{q}}[4]_{\sqrt{q}}}{[3]_{\sqrt{q}}[5]_{\sqrt{q}}} \cdot \frac{[6]_{\sqrt{q}}[6]_{\sqrt{q}}}{[5]_{\sqrt{q}}[7]_{\sqrt{q}}} \cdots .
$$


If we define the $q$-deformed sine function $\operatorname{asin}_{q}(x)=\frac{1}{2 i}\left(e_{q}(i z)-e_{q}(-i x)\right)$ and the $q$-analogue of $\pi, \pi_{q}$, as

$$
\sin _{q}\left(\pi_{q} x\right)=\pi_{q}[x] \prod_{k=1}^{\infty}\left[1+\frac{x}{k}\right]_{q^{k}}\left[1-\frac{x}{k}\right]_{q^{k}},
$$

then we have $\sin _{q}\left(\frac{\pi_{q}}{2}\right)=1$ and $\sin _{q}\left(n \pi_{q}\right)=0$ for all $n \in \mathbb{Z}$. The $q$-analogue of Wallis formula implies

$$
\Gamma_{q}\left(\frac{1}{2}\right)=\sqrt{\pi_{q}}
$$

By (4) and (5) we obtain the following result (see [4]).

Proposition 2.3. We have

$$
\Gamma_{q}(x) \Gamma_{q}(1-x)=\frac{\pi_{q}}{\sin _{q}\left(\pi_{q} x\right)} .
$$

Another definition of the $q$-deformed Gamma function is given by $q$-analogue of Weierstrass product formula:

$$
\frac{1}{\Gamma_{q}(x)}=[x] e^{\gamma_{q} x} \prod_{k=1}^{\infty}\left[1+\frac{x}{k}\right]_{q^{k}} e^{-\frac{x}{[k]}},
$$

where the $q$-analogue of the Euler-Mascheroni constant $\gamma_{q}$ is defined by

$$
\gamma_{q}=\lim _{n \rightarrow \infty}\left(\frac{1}{[1]}+\frac{1}{[2]}+\cdots+\frac{1}{[n]}-\ln [n]\right) .
$$

Now, let us extend our work to the $q$-deformed digamma function and polygamma function. From (1), we have

$$
\ln \Gamma_{q}(x+1)=\lim _{n \rightarrow \infty}\left(\ln [n] !+x \ln [n]-\sum_{k=1}^{n}[x+k]\right) .
$$

Then the digamma function $F_{q}(x)$ is defined as follows:

$$
F_{q}(x)=\frac{d \ln \Gamma_{q}(x+1)}{d x}=-\gamma_{q}+\sum_{k=1}^{\infty}\left(\frac{1}{[k]}+\frac{q^{x+k} \ln q}{1-q^{x+k}}\right) .
$$

If we insert $x=0$ into (6), we have

$$
F_{q}(0)=-\gamma_{q}+\sum_{k=1}^{\infty} \frac{1-q+q^{k} \ln q}{1-q^{k}} .
$$

It can be easily checked that

$$
\lim _{q \rightarrow 1} F_{q}(0)=-\gamma=-0.577216 \cdots
$$


Differentiating the $q$-deformed digamma function repeatedly, we obtain the $q$ deformed polygamma function as follows:

$$
F_{q}^{(n)}(x)=\frac{d^{n+1} \ln \Gamma_{q}(x+1)}{d x^{n+1}}=(\ln q)^{n+1} \sum_{k=1}^{\infty} \frac{\sum_{l=1}^{n-1} a_{l}^{(n)} q^{l(x+k)}}{\left(1-q^{x+k}\right)^{n+1}}
$$

where $a_{l}^{(n+1)}=l a_{l}^{(n)}+(n-l+2) a_{l-1}^{(n)}, l=2,3, \ldots, n-2$, and $a_{1}^{(n)}=a_{n}^{(n)}=1$. Note that $a_{l}^{n}$ is the Eulerian number which is given by $a_{l}^{n}=\sum_{j=0}^{l}(-1)^{j}(l-$ $j)^{n}\left(\begin{array}{c}n+1 \\ j\end{array}\right)($ see $[16])$.

\section{Representation of $q$-oscillator algebra in terms of the $q$-factorial polynomial}

In this section, we discuss the representation of a $q$-oscillator algebra by introducing $q$-factorial polynomials which are derived from the $q$-gamma function. Let us define the $q$-factorial polynomial as follows

$$
\phi_{n}(x)=\frac{\Gamma_{q}(x+1)}{\Gamma_{q}(x+1-n)}=\prod_{j=0}^{n-1}[x-j] .
$$

The $q$-factorial polynomial behaves as ordinary monomials under the action of the operators $\hat{a}^{\dagger}=x e^{-\partial_{x}}$ and $\hat{a}=q^{-x-1}\left(e^{\partial_{x}}-1\right)$, where $\partial_{x}=\frac{d}{d x}$ is an ordinary derivative. Indeed, $\hat{a}$ and $\hat{a}^{\dagger}$ are called step operators when they appear in the $q$-deformed quantum theory.

Proposition 3.1. The step operators satisfy $\hat{a}^{\dagger} \phi_{n}(x)=\phi_{n+1}(x)$ and $\hat{a} \phi_{n}(x)=$ $q^{-n}[n] \phi_{n-1}(x)$.

Proof. Acting $\hat{a}^{\dagger}$ on $\phi_{n}(x)$, we have

$\hat{a}^{\dagger} \phi_{n}(x)=x \phi_{n}(x-1)=x \prod_{j=0}^{n-1}[x-(j+1)]=x \prod_{j=1}^{n}[x-j]=\prod_{j=0}^{n}[x-j]=\phi_{n+1}(x)$.

Acting $\hat{a}$ on $\phi_{n}(x)$, we have

$$
\begin{aligned}
\hat{a} \phi_{n}(x) & =q^{-x-1}\left[\phi_{n}(x+1)-\phi_{n}(x)\right]=q^{-x-1}\left(\prod_{j=0}^{n-1}[x-(j-1)]-\prod_{j=0}^{n-1}[x-j]\right) \\
& =q^{-x-1}\left([x+1] \prod_{j=1}^{n-1}[x-(j-1)]-[x-(n-1)] \prod_{j=0}^{n-2}[x-j]\right) \\
& =q^{-x-1}\{[x+1]-[x-(n-1)]\} \prod_{j=0}^{n-2}[x-j] \\
& =q^{-n}[n] \prod_{j=0}^{n-2}[x-j]=q^{-n}[n] \phi_{n-1}(x),
\end{aligned}
$$


which completes the proof.

Moreover, the step operators satisfy the $q$-oscillator algebra:

$$
\hat{a} \hat{a}^{\dagger}-q^{-1} \hat{a}^{\dagger} \hat{a}=q^{-1} \text {. }
$$

Acting $\hat{a}^{\dagger} \hat{a}$ on the $q$-factorial polynomial $\phi_{n}(x)$ yields the difference equation

$$
\hat{a}^{\dagger} \hat{a} \phi_{n}(x)=q^{-n}[n] \phi_{n}(x)
$$

which can be written as

$$
\left(q^{-n}[n]+x q^{-x}\right) \phi_{n}(x)=x q^{-x} \phi_{n}(x-1) .
$$

Now let us investigate the eigenfunction and eigenvalue of the position operator defined by $\hat{X}=\hat{a}+\hat{a}^{\dagger}$. If we denote the eigenfunction and eigenvalue of the position operator by $\psi(x)$ and $x$, respectively, then we have $\hat{X} \psi(x)=x \psi(x)$ or

$$
\left[x e^{-\partial_{x}}+q^{-x-1}\left(e^{\partial_{x}}-1\right)\right] \psi(x)=x \psi(x) .
$$

Then, (7) can be rewritten as

$$
\psi(x+1)-\psi(x)=x q^{x+1}(\psi(x)-\psi(x-1)) .
$$

If we set $g(x)=\psi(x)-\psi(x-1)$, we have $g(x+1)=x q^{x+1} g(x)$. The transform $g(x)=q^{x(x+1) / 2} h(x)$ gives $h(x+1)=x h(x)$. Hence, $h(x)=\prod_{j=1}^{\infty}(x-j)$. Then the eigenfunction of the position operator satisfies the following recurrence relation

$$
\psi(x)-\psi(x-1)=q^{x(x+1) / 2} \prod_{j=1}^{\infty}(x-j) .
$$

The solution of (8) is given by

$$
\psi(x)=\sum_{n=0}^{\infty} q^{-(n-1) x+\frac{(n-1)(n-2)}{2}} \prod_{k=n}^{\infty}(x-k) .
$$

Acknowledgements. The authors would like to express their gratitude for the valuable comments and suggestions of referees. The present Research has been conducted by the Research Grant of Kwangwoon University in 2014.

\section{References}

[1] M. Arik and D. Coon, Hilbert spaces of analytic functions and generalized coherent states, J. Mathematical Phys. 17 (1976), no. 4, 524-527.

[2] R. Askey, The q-gamma and q-beta functions, Appl. Anal. 8 (1978), no. 2, 125-141.

[3] N. M. Atakishiyev, A. Frank, and K. B. Wolf, A simple difference realization of the Heisenberg q-algebra, J. Math. Phys. 35 (1994), no. 7, 3253-3260.

[4] R. W. Gosper, Experiments and Discoveries in q-Trigonometry, In Symbolic Computation, Number Theory, Special Functions, Physics and Combinatorics, Proceedings of the Conference Held at the University of Florida, Gainesville, FL, 1999.

[5] F. H. Jackson, A generalization of the functions $\Gamma(n)$ and $x^{n}$, Proc. Roy. Soc. London. 74 (1904), 64-72. 
[6] The basic gamma function and the elliptic functions, Proc. Roy. Soc. London. A 76 (1905), 127-144.

[7] T. Kim, q-Volkenborn integration, Russ. J. Math. Phys. 9 (2002), no. 3, 288-299.

[8] T. Kim and C. Adiga, On the q-analogue of gamma functions and related inequalities, J. Inequal. Pure Appl. Math. 6 (2005), no. 4, Article 118, 4 pp.

[9] A. Macfarlane, On q-analogues of the quantum harmonic oscillator and the quantum group $S U(2)_{q}$, J. Phys. A 22 (1989), no. 21, 4581-4588.

[10] M. Mansour, An asymptotic expansion of the q-gamma function $\Gamma_{q}(x)$, J. Nonlinear Math. Phys. 13 (2006), no. 4, 479-483.

[11] T. Mansour and A. Shabani, Some inequalities for the q-digamma function, J. Inequal. Pure Appl. Math. 10 (2009), no. 1, Article 12, 8 pp.

[12] S.-H. Rim and T. Kim, A note on the q-analogue of p-adic log-gamma function, Adv. Stud. Contemp. Math. 18 (2009), no. 2, 245-248.

[13] A. Salem, An infinite class of completely monotonic functions involving the q-gamma function, J. Math. Anal. Appl. 406 (2013), no. 2, 392-399.

[14] — A completely monotonic function involving q-gamma and q-digamma functions, J. Approx. Theory 164 (2012), no. 7, 971-980.

[15] W. T. Sulaiman, Some inequalities for the q-digamma functions, J. Concr. Appl. Math. 10 (2012), no. 3-4, 301-308.

[16] N. J. Sloane, The On-Line Encyclopedia of Integer Sequences, http://oeis.org, 2010.

[17] J. Thomae, Beitrage zur Theorie der durch die Heinesche Reihe, J. Reine Angew. Math. 70 (1869), 258-281.

Won SANG Chung

Department of Physics

Gyeongsang National University

JiNJU 660-701, KoREA

E-mail address: mimip4444@hanmail.net

TAEKYUN KIM

Department of Mathematics

KWANGWOON UNIVERSITY

Seoul 139-701, Korea

E-mail address: tkkim@kw.ac.kr

Toufik Mansour

Department of Mathematics

UNIVERSITY OF HAIFA

3498838 HAIFA, ISRAEL

E-mail address: tmansour@univ.haifa.ac.il 